\title{
INDICADORES FECAIS DE BOVINOS NELORE ALIMENTADOS COM DIETAS DE ALTA PROPORÇÃO DE CONCENTRADO
}

\author{
HÉlio LOUREdo da Silva ${ }^{1}$, Aldi Fernandes de SOUZA FranÇA ${ }^{1}$, Flávio GERALdo Castro \\ FERREIRA $^{2}$, ÉDER DE SOUSA FERNANDES ${ }^{3}$, ALINE LANDIM $^{4}$, EDUARDO RODRIGUES CARVALHO ${ }^{5}$ \\ ${ }^{1}$ Professores Doutores da Escola de Veterinária e Zootecnia da Universidade Federal de Goiás, Goiânia, GO, Brasil - \\ heliolou@yahoo.com.br \\ ${ }^{2}$ Diretor Técnico da Agrocria Produtos Veterinários, Goiânia, GO, Brasil \\ ${ }^{3}$ Técnico do Laboratório de Nutrição animal da Escola de Veterinária e Zootecnia da UFG, Goiânia, GO, Brasil \\ ${ }^{4}$ Pós-graduanda em Ciência Animal da Universidade Federal de Goiás, Goiânia, GO, Brasil \\ ${ }^{5}$ Professor Doutor do Instituto Federal de Educação, Ciência e Tecnologia Goiano, Iporá, GO, Brasil.
}

\begin{abstract}
O presente trabalho teve como objetivo avaliar os efeitos de dietas de alta proporção de concentrado sobre características fecais em 20 bovinos Nelore machos com idade de 28 meses, em delineamento inteiramente casualizado. Os tratamentos foram constituídos de dieta total mais bagaço in natura - DT+BIN $(54,52 \%$ de sorgo moído, 10,94\% de caroço de algodão, $18 \%$ de casca de soja, $2,54 \%$ de farelo de soja, $10 \%$ bagaço de cana in natura e $4 \%$ de núcleo farelado), milho grão inteiro - MGI (75\% de milho grão inteiro, $10 \%$ de casca de soja e $15 \%$ de núcleo peletizado) e dieta total - DT $(44,44 \%$ de sorgo moído, $16,70 \%$ de caroço de algodão, $28,86 \%$ de casca de soja e $10 \%$ de núcleo farelado). Para determinação do $\mathrm{pH}$ das fezes e do amido fecal, amostras de fezes foram coletadas do reto de cada bovino nos dias 54, 55, 56 e 57 de experimento no período da manhã. $\mathrm{O}$ pH fecal foi determinado após adição de $100 \mathrm{~mL}$ de água destilada deionizada em $15 \mathrm{~g}$ de fezes frescas úmidas com a introdução da ponta do eletrodo de um peagâmetro microprocessado. O restante da amostra foi colocado em gelo, para depois ser congelado. Com o intuito de avaliar ocorrências de distúrbios gastrintestinais, as fezes frescas dos animais nas baias experimentais individuais foram avaliadas diariamente durante todo o experimento em três períodos. Os valores médios em percentagem do amido
\end{abstract}

PALAVRAS-CHAVE: amido; confinamento; fezes; ração. fecal, matéria seca fecal, medida de $\mathrm{pH}$ para o local de fermentação do amido e consumo de amido/kg não foram influenciados $(\mathrm{P}>0,05)$ pelos tratamentos. A FDN das fezes e o escore de consistência fecal foram influenciados pelos tratamentos $(\mathrm{P}<0,05)$. A dieta MGI com menor teor de FDNfe apresentou menor escore de fezes e menor percentagem de FDN fecal. Porém, a percentagem de amido e $\mathrm{pH}$ fecal, MS fecal e o consumo do amido não foram afetados pelo teor de FDNfe nas rações. A concentração de FDN fecal foi igual para os tratamentos DT+BIN e DT. O menor teor de FDN fecal no tratamento MGI é função do menor consumo de FDN pelos animais e, possivelmente, da maior digestibilidade dessa dieta. Os animais do tratamento MGI apresentaram fezes com consistência mais mole, com o valor de escore de 2,92. As fezes dos bovinos do tratamento DT+BIN foram de consistência mais firme com valor de escore de 3,12, considerada normal, e a dos animais do tratamento DT foram de consistência mais dura com valor de escore de 3,20 . Houve uma relação consistente positiva entre amido fecal e eficiência alimentar e uma tendência de relação positiva entre amido fecal e GMD. Dieta de alta proporção de concentrado com adição de $10 \%$ de BIN na matéria seca proporciona maior freqüência de fezes com escore de consistência firme.

\section{FECAL INDICATORS OF NELORE BOVINES FED HIGH CONCENTRATE DIETS}

\section{ABSTRACT}

The present work had as objective to evaluate the effects of high grain diets on fecal parameters in 20 Nellore bovines at 28 months of age. The experimental design was completely randomized. Treatments were constituted of the following diets: total mixed ration + sugarcane bagasse DT + BIN (10\% in natura sugarcane bagasse, 
$54.52 \%$ ground sorghum, $10.94 \%$ cottonseed, $18 \%$ soybean hull, $2.54 \%$ soybean meal, and $4 \%$ premix); whole corn grain MGI $(75 \%$ whole corn grain, $10 \%$ soybean hull, and $15 \%$ premix) and total mixed ration DT (44.41\% ground sorghum, $16.7 \%$ cottonseed, $28.89 \%$ soybean meal, and $10 \%$ premix). For the assessment of feces $\mathrm{pH}$ and fecal starch, samples of feces were taken from the rectum of each animal on the $54^{\text {th }}, 55^{\text {th }}, 56^{\text {th }}$ and $57^{\text {th }}$ days of the experiment in the morning. Fecal $\mathrm{pH}$ was determined after the addition of $100 \mathrm{~mL}$ of distilled water in $15 \mathrm{~g}$ of fresh feces by the introduction of the tip of the electrode of a microprocessed $\mathrm{pH}$ meter. The remaining sample was stored in ice for later freezing. Fresh feces of the animals were evaluated daily during the whole experimental length in three periods aiming at evaluating occurrences of gut disturbances. Percentage means of fecal starch, fecal dry matter, $\mathrm{pH}$ in the site of starch fermentation and starch intake were not influenced by treatments $(\mathrm{P}>0.05)$. NDF of feces and score of fecal consistency were influenced by treatments $(\mathrm{P}<0.05)$. The

KEYWORDS: diet; feces; feedlot; starch.

\section{INTRODUÇÃO}

Dos nutrientes existentes na dieta de bovinos intensivamente alimentados, o amido é o maior componente e o que fornece a maior quantidade de energia digestível consumida pelo animal. Então, a avaliação da perda de amido como resultado de problemas de manejo e digestibilidade, causada pelas diversas formas de processamento de grão, seria uma medida de bom impacto para melhoria no desempenho de bovinos confinados.

Métodos de processamento para reduzir o tamanho da partícula ou alterar a matriz proteica que cimenta grânulos de amido, incrementariam a extensão da digestão no rúmen e no intestino delgado. Dados de desempenho de bovinos em crescimento alimentados com grãos de milho e sorgo processados indicaram que o amido usado foi $42 \%$ mais eficientemente, se digerido de preferência no intestino delgado e não no rúmen (OWENS et al., 1986).

Por conseguinte, é necessário predizer o fluxo e desaparecimento do amido no intestino delgado no momento de formular uma dieta. Baseados em métodos atuais, meios adequados e confiáveis de avaliações da digestão no intestino delgado não estão disponíveis. Sem a habilidade para precisamente descrever e predizer a digestão do amido no intestino delgado não se consegue otimizar a eficiência digestiva do amido. Pode-se somente esperar um resultado, evitando-se a excreção fecal do amido, pelo processamento do grão e manejo alimentar (HUNTINGTON et al., 2006).

LEDOUX et al. (1985) demonstraram que o
MGI diet (with lowest content of peNDF) presented the lowest score of feces and percentage of fecal NDF. However, the percentage of starch and fecal $\mathrm{pH}$, fecal dry matter and starch intake were not affected by peNDF contents in the diets. Animals fed MGI had lower fecal NDF. The concentration of fecal NDF was similar for the treatments DT + BIN and DT. The lower content of fecal NDF in the treatment MGI is due to lower intake of NDF and possibly because of better digestibility of this diet. Animals in MGI treatment presented feces with softer consistence (score 2.92). Feces of the animals in DT + BIN treatment were more consistent (score 3.12), and the ones from the animals DT + BIN treatment DT were even harder (score 3.2). There was positive correlation between fecal starch and intake efficiency and a tendency of positive correlation between fecal starch and GMD. Diets with high concentrate proportion with the addition of $10 \%$ BIN in dry matter produces a higher frequency of consistent feces.

$\mathrm{pH}$ fecal e ruminal não foram influenciados pelos níveis de feno. Ao contrário, o desempenho dos animais e o pH das fezes não aumentaram à medida que o nível de feno subiu de 4 para $24 \%$ na dieta. Posto que o consumo se manteve constante em 1,7\% do peso corporal no ensaio de metabolismo, esse dado sugere que o consumo do amido de milho não foi suficientemente alto para diminuir o $\mathrm{pH}$ fecal. Por outro lado, RUSSELL et al. (1981) informaram uma alta correlação entre o $\mathrm{pH}$ fecal e o consumo de amido em novilhos alimentados com dietas de alto concentrado.

TURGEON et al. (1983) concluíram que a percentagem de amido fecal e $\mathrm{pH}$ fecal não foram influenciados pelo tamanho da partícula. $\mathrm{O}$ amido fecal diminuiu linearmente conforme o nível da forragem aumentou na dieta. Uma relação negativa entre amido fecal e $\mathrm{pH}$ fecal $(\mathrm{r}=-0,42)$ foi observada. Entretanto, nem o amido fecal nem $\mathrm{pH}$ fecal foram altamente correlacionados ao desempenho animal.

LEE et al. (1982) observaram que a correlação entre $\mathrm{pH}$ e amido fecal foi de $-0,86,-0,34$, $-0,31$ e $-0,54$ para $56,84,112$ e 140 dias do período de alimentação, respectivamente, ocorrendo correlação significativa somente para 56 dias. O amido fecal tendeu a ser maior à medida que a proporção de MGI foi aumentada na dieta. Em geral, houve uma tendência para $\mathrm{pH}$ fecal mais alto ser associado com baixo conteúdo de amido fecal. Portanto, $\mathrm{pH}$ fecal pode ser um indicador útil de avaliação do amido geral em ruminantes alimentados com dietas de alto concentrado, porém, não em todos os casos. 
O objetivo deste trabalho foi avaliar os efeitos de dietas de alta proporção de concentrado sobre os indicadores fecais de bovino Nelore macho em confinamento.

\section{MATERIAL E MÉTODOS}

O experimento foi desenvolvido no Setor de Bovinocultura da Fazenda Barreiro, situada no Município de Silvânia - Goiás com $16^{\circ} 29^{\prime} 50,05^{\prime \prime}$ de altitude e $48^{\circ} 47^{\prime} 31,44^{\prime \prime}$ de longitude e altitude de $1000 \mathrm{~m}$. O município apresenta clima tropical com chuvas estacionais bem distribuídas de outubro a março. O trabalho foi realizado entre os dias 9 de dezembro de 2007 e 27 de março de 2008. A temperatura média mensal durante o período experimental foi de $24,23^{\circ} \mathrm{C}$, com precipitação pluviométrica mensal média de $279,08 \mathrm{~mm}$ e umidade relativa média do ar de $74,25 \%$. As avaliações laboratoriais foram realizadas nos Laboratórios de Análises de Alimentos da Escola de Veterinária e Zootecnia da UFG.

Foram utilizados 20 animais inteiros da raça Nelore com idade de 28 meses e peso vivo médio de $336,61 \mathrm{~kg}$ no inicio do período experimental. Os animais foram sorteados aleatoriamente nos tratamentos e adaptados às instalações, ao manejo e ao consumo das dietas durante 21 dias. A adaptação dos animais às dietas experimentais, ração mistura total (TMR), foi por meio de regime alimentar restrito, com o fornecimento de $1,3 \%$ da dieta em relação ao peso vivo na matéria natural, no primeiro dia, e partir daí procedeu-se o aumento de $10 \%$ na dieta diariamente até o final da adaptação. A duração total do experimento foi de 105 dias em regime de confinamento, com pesagens, após jejum de sólidos, a intervalos médios de 21 dias.

$\mathrm{O}$ delineamento aplicado foi o inteiramente ao acaso, com três tratamentos: dieta total + bagaço de cana in natura (DT+BIN, $\mathrm{n}=7$ ), milho grão inteiro (MGI, n=6) e dieta total (DT, $n=7$ ). Os animais foram distribuídos aleatoriamente em baias individuais $\left(12 \mathrm{~m}^{2}\right)$, cimentadas $\left(7 \mathrm{~m}^{2}\right)$, parcialmente cobertas $\left(7 \mathrm{~m}^{2}\right)$ e providas de comedouro e bebedouro (torneira com bóia) de concreto. Antes do inicio do experimento, os animais foram identificados com brincos numerados, pesados, vacinados e submetidos a controle de ecto- e endoparasitas.

TABELA 1 - Relação dos níveis de garantia e composição química dos núcleos incluídos nas dietas experimentais

\begin{tabular}{lccc}
\hline \multirow{2}{*}{ Níveis de garantia } & Núcleo farelado & Núcleo peletizado & Núcleo farelado \\
\cline { 2 - 4 } & DT + BIN & MGI & DT \\
\cline { 2 - 4 } MS $(\%)$ & \multicolumn{3}{c}{ Valores } \\
NDT $(\%)$ & 90,0 & 90,0 & 90,0 \\
PB (\%) & 25,0 & 64,4 & 64,0 \\
NÑ̃ Eq. Prot $(\%)$ & 78,0 & 44,5 & 27,7 \\
Fibra bruta (\%) & 62,50 & 9,40 & 2,30 \\
Cálcio $(\mathrm{g} / \mathrm{kg})$ & 2,0 & 7,50 & 13,0 \\
Fósforo $(\mathrm{g} / \mathrm{kg})$ & 110 & 29,35 & 44,0 \\
Enxofre $(\mathrm{g} / \mathrm{kg})$ & 22,0 & 5,87 & 8,8 \\
Magnésio $(\mathrm{g} / \mathrm{kg})$ & 9,0 & 2,40 & 3,60 \\
Potássio $(\mathrm{g} / \mathrm{kg})$ & 2,50 & 0,67 & 1,0 \\
Sódio $(\mathrm{g} / \mathrm{kg})$ & 28,0 & 3,0 & 3,0 \\
Cobalto $(\mathrm{mg} / \mathrm{kg})$ & 34,75 & 12,01 & 12,0 \\
Cobre $(\mathrm{mg} / \mathrm{kg})$ & 15,0 & 4,0 & 6,0 \\
Cromo $(\mathrm{mg} / \mathrm{kg})$ & 500 & 133,4 & 200 \\
Iodo $(\mathrm{mg} / \mathrm{kg})$ & 3,5 & 0,93 & 1,40 \\
Manganês $(\mathrm{mg} / \mathrm{kg})$ & 12,50 & 3,34 & 5,0 \\
Molibdênio $(\mathrm{mg} / \mathrm{kg})$ & 500 & 133,40 & 200 \\
Níquel $(\mathrm{mg} / \mathrm{kg})$ & 1,0 & 0,27 & 0,40 \\
Selênio $(\mathrm{mg} / \mathrm{kg})$ & 1,0 & 0,27 & 0,40 \\
Zinco $(\mathrm{mg} / \mathrm{kg})$ & 5,0 & 1,32 & 2,0 \\
Monensina $(\mathrm{mg} / \mathrm{kg})$ & 750 & 200,10 & 300 \\
Levedura $(\mathrm{g} / \mathrm{kg})$ & 750 & 200,10 & 300 \\
Vitamina A $(\mathrm{UI} / \mathrm{kg})$ & 12,50 & 3,34 & 5,0 \\
Vitamina D $(\mathrm{UI} / \mathrm{kg})$ & 55.000 & 14.674 & 22.000 \\
Vitamina E $(\mathrm{UI} / \mathrm{kg})$ & 8.500 & 2.267 & 3.400 \\
\hline & 400 & 106,72 & 160 \\
\hline
\end{tabular}


As dietas foram compostas de milho grão inteiro (MGI), sorgo moído (SM), caroço de algodão (CA), farelo de soja (FS), casca de soja (CS), bagaço in natura de cana-de-açúcar (BIN) e núcleos específicos para cada dieta, a fim de atender às exigências mineral e proteica dos animais (Tabelas 1 e 2).

TABELA 2 - Composição químico-bromatológica dos alimentos utilizados nas dietas experimentais na base da matéria seca (\% MS) fornecidas aos animais

\begin{tabular}{lcccccc}
\hline Alimentos & MS & PB & FDA & FDN & MM & EE \\
\hline Milho & 88,64 & 7,86 & 4,31 & 7,30 & 1,86 & 3,09 \\
Sorgo & 89,34 & 7,21 & 4,03 & 8,41 & 1,34 & 1,54 \\
Caroço de algodão & 89,55 & 23,10 & 28,34 & 41,38 & 6,20 & 16,13 \\
Farelo de soja & 87,76 & 46,60 & 10,42 & 21,30 & 6,55 & 2,34 \\
Casca de soja & 92,35 & 10,91 & 40,52 & 64,34 & 3,38 & 0,92 \\
Bagaço de cana in natura & 74,8 & 1,71 & 56,1 & 74,53 & 1,25 & - \\
\hline Faga
\end{tabular}

Fonte: valores analisados LANA/DPA/EV/UFG. MS: matéria seca; PB: proteína bruta; FDA: fibra em detergente ácido; FDN: fibra em detergente neutro; MM: matéria mineral; EE: extrato etéreo.

Os tratamentos foram constituídos por rações médio de 1,40 kg/dia, conforme o NRC (1996), completas e isoproteicas, calculadas para atenderem sendo calculadas pelo sistema (CNCPS de as exigências de bovinos de corte para um ganho CORNELL, 2002) (Tabela 3).

TABELA 3 - Composição centesimal das dietas experimentais, dieta total mais bagaço in natura (DT+BIN), milho grão inteiro (MGI) e dieta total (DT) na matéria seca (\% MS)

\begin{tabular}{lccc}
\hline Alimentos & \multicolumn{3}{c}{ Tratamentos } \\
\cline { 2 - 4 } & DT+BIN & MGI & DT \\
\hline Caroço de algodão & 10,94 & & 16,70 \\
Casca de soja & 18,00 & 10,00 & 28,86 \\
Sorgo moído & 54,52 & & 44,44 \\
Núcleo farelado & 4,0 & & \\
Milho grão inteiro & & 75,00 & \\
Núcleo peletizado & & 15,00 & \\
Bagaço de cana in natura & 10,00 & & \\
Farelo de soja & 2,54 & & \\
\hline
\end{tabular}

A composição bromatológica das dietas experimentais está descrita na Tabela 4. A virginiamicina (10\% da Phibro Animal Health®) foi misturada à parte na ração, na dosagem de $150 \mathrm{mg} /$ $\mathrm{cab} / \mathrm{dia}$.

TABELA 4 - Composição química das dietas experimentais, dieta total mais bagaço in natura (DT + BIN), de milho grão inteiro (MGI), dieta total (DT) em percentagem na matéria seca (\%MS)

\begin{tabular}{lccc}
\hline \multicolumn{1}{c}{ NUTRIENTES } & \multicolumn{3}{c}{ Tratamentos } \\
\cline { 2 - 4 } & DT+BIN & MGI & DT \\
\hline Matéria seca $^{1}$ & 88,00 & 88,00 & 88,00 \\
Proteína bruta $^{1}$ & 12,03 & 12,30 & 12,33 \\
Nutrientes digestíveis totais $^{2}$ & 74,68 & 81,21 & 80,41 \\
Fibra detergente neutro $^{1}$ & 42,10 & 32,95 & 39,04 \\
Fibra detergente ácido $^{1}$ & 25,15 & 16,03 & 21,81 \\
Fibra detergente neutro fisicamente efetivo $^{3}$ & 33,5 & 8,17 & 28,77 \\
Extrato etéreo $^{1}$ & 3,34 & 2,88 & 4,81 \\
Carboidrato não fibroso $^{4}$ & 38,56 & 48,48 & 37,98 \\
Amido $^{1}$ & 39,78 & 54,62 & 48,70 \\
Minerais $^{1}$ & 4,57 & 3,39 & 5,28 \\
\hline IVar $^{1}$ & &
\end{tabular}

1Valores analisados LANA/DPA/EVZ/UFG; ${ }^{2}$ Valores de tabela; ${ }^{3}$ MERTENS (1997); ${ }^{4}$ SNIFFEN et al. (1992). 
O consumo voluntário das dietas e dos nutrientes foi determinado mediante diferenças entre $o$ oferecido e as sobras. As dietas, depois de pesadas, foram distribuídas na forma de dieta mistura total em duas refeições diárias, às $8 \mathrm{~h}$ e $17 \mathrm{~h}$, permitindo-se sobras de aproximadamente $5 \%$ do ofertado. As amostras das dietas oferecidas e das sobras, após pesagem de controle diário foram coletadas duas vezes por semana, para análise laboratorial. O teor de MS das dietas foi analisado semanalmente e as rações experimentais ajustadas em função dos resultados. As pesagens dos animais foram realizadas no início do período de adaptação, no início do período experimental e a cada 21 dias, sempre em jejum hídrico e alimentar de 14 horas.

A avaliação do tamanho das partículas foi realizada pelo separador de partícula do Estado da Pensilvânia (PSPS). Para determinar o tamanho médio de partículas das amostras das dietas oferecidas aos animais e das sobras coletadas uma vez em cada período experimental, utilizou-se um conjunto de três peneiras com orifícios de $19 \mathrm{~mm}, 8 \mathrm{~mm}$ e $1,18 \mathrm{~mm}$ e um fundo de caixa fechado. A caixa com a amostra foi agitada $1,1 \mathrm{~Hz}$ (1,1 ciclo por segundo) no total de 66 ciclos por minuto a uma distância de $17 \mathrm{~cm}$. Realizada sistematicamente sempre pelo mesmo operador, a amostra foi segregada em quatro estratos de diferentes tamanhos: acima de $19 \mathrm{~mm}$, entre 19 e $8 \mathrm{~mm}$, entre 8 e $1,18 \mathrm{~mm}$ e inferiores a $1,18 \mathrm{~mm}$. O cálculo da porcentagem de partículas retidas em cada peneira foi realizado de forma direta, considerando o somatório dos pesos da fração retida em cada peneira, descontando-se a tara da peneira. O cálculo ponderal do tamanho médio das partículas foi dado pelo tamanho médio das partículas retidas em cada peneira e o percentual de retenção em relação ao peso total da amostra estratificada conforme (KONONOFF, 2003). Os cálculos da FDNfe foram obtidos de acordo com MERTENS (1997).

Para determinação do $\mathrm{pH}$ das fezes e do amido fecal, amostras fecais foram coletadas do reto de cada bovino no período da manhã, entre os horários das 9:30 às 12:00 horas, nos dias 54, 55, 56 e 57 do experimento. $\mathrm{O} \mathrm{pH}$ fecal foi determinado após adição de $100 \mathrm{~mL}$ de água destilada deionizada em $15 \mathrm{~g}$ de fezes frescas úmidas com a introdução da ponta do eletrodo de um peagâmetro microprocessado. $\mathrm{O}$ restante da amostra foi colocado em gelo, para depois ser congelado (TURGEON, 1983).

Antes das análises, as amostras fecais foram descongeladas e feitas amostras compostas dos quatro dias de coleta para cada animal as quais, em seguida, foram secas em estufa com circulação de ar forçada a $65^{\circ} \mathrm{C}$ (MS) e moídas em moinho do tipo Willey com peneira de $1 \mathrm{~mm}$.

Com o intuito de avaliar as ocorrências de Ci. Anim. Bras., Goiânia, v.13, n2, p. 145-156, abr./jun. 2012 distúrbios gastrintestinais, as fezes frescas dos animais nas baias experimentais, foram avaliadas diariamente durante todo $\mathrm{o}$ experimento em três períodos consecutivos (janeiro, fevereiro e março) subjetivamente por uma pessoa treinada considerandose quatro escores visuais. A medida de consistência fecal foi determinada por escore visual, sendo: $1=$ líquida: consistência líquida com som de respingo no contato com a água e que se espalha facilmente com o impacto no solo; 2 = mole: fezes soltas; respinga moderadamente e difusamente no impacto com o solo fazendo o som de respingo de um objeto em contato com a água; 3 = firme: mas não dura, amontoada, porém, pastosa e ligeiramente dispersa e assentada no impacto com o solo; 4 = dura: aparência dura, forma original não alterada e assentada no impacto com o solo (IRELANPPERRY \& STALLINGS 1993).

Avaliação químico-bromatológica da matéria seca (MS), matéria orgânica (MO), proteína bruta (PB), amido, extrato etéreo (EE) e mineral das dietas fornecidas, sobras e fezes foi realizada no laboratório de análises bromatológicas do Departamento de Produção Animal da Escola de Veterinária e Zootecnia da UFG. As amostras foram pré-secas em estufa de ventilação forçada a $65^{\circ} \mathrm{C}$ e a MS determinada em estufa a $105^{\circ} \mathrm{C}$. As determinações da PB (método de Kjeldhal), EE e minerais das rações (oferecidas e sobras) e fezes foram efetuadas de acordo com A.O.A.C. (1990). A fibra em detergente neutro (FDN) e a fibra em detergente ácido (FDA) foram calculadas pelo método sequencial de (ROBERTSON \& VAN SOEST, 1981).

A determinação do amido das rações (oferecidas e sobras) e fezes foi efetuada pelo método enzimático segundo metodologia descrita por (CAMPOS et al., 2004).

$\mathrm{O}$ modelo estatístico adotado foi: $\mathrm{Y}_{\mathrm{ijk}}=\mathrm{m}+\mathrm{T}_{\mathrm{i}}$ $+E_{\mathrm{ijk}}$, em que: $Y_{\mathrm{ijk}}=$ valor observado para a característica analisada; $\mathrm{m}=$ média geral; $\mathrm{T}_{\mathrm{i}}=$ efeito da dieta completa de alta proporção de concentrado $\mathrm{i}$; e $\mathrm{E}_{\mathrm{ijk}}$ = erro aleatório comum a todas as observações.

Os resultados foram analisados usando o procedimento PROC GLM do SAS (2000), aplicandose o teste de Tukey a 5\% de probabilidade, para comparação das médias. Foi utilizada a raiz quadrada para estabilizar os dados de variância para os parâmetros de consistência de fezes, firme, dura mole e líquida. Para a análise do escore de fezes foi utilizado o teste de Kruskal-Wallis. Os resultados foram submetidos à análise de correlação de Pearson pelo procedimento CORR.

\section{RESULTADOS E DISCUSSÃO}

Os valores médios em percentagem do amido fecal, matéria seca fecal, medida de $\mathrm{pH}$ para o local de 
fermentação do amido, consumo de amido $/ \mathrm{kg}$, ganho médio diário e a eficiência alimentar não foram influenciados $(\mathrm{P}>0,05)$ pelos tratamentos (Tabela 5).
O escore de consistência fecal e a FDN das fezes foram influenciados $(\mathrm{P}<0,05)$ pelos tratamentos.

TABELA 5 - Efeitos de dietas de alta proporção de concentrado nos conteúdos de MS fecal, consumo de amido, amido fecal (\% MS), FDN fecal (\% MS), pH fecal, escore de consistência fecal e ganho médio diário $(\mathrm{kg} / \mathrm{dia})$ e eficiência alimentar $(\mathrm{kg} / \mathrm{kg})$

\begin{tabular}{lcccc}
\hline \multirow{2}{*}{\multicolumn{1}{c}{ Variáveis/Items }} & \multicolumn{3}{c}{ Tratamentos } & \multirow{2}{*}{ CV \% } \\
\cline { 2 - 4 } & DT+BIN & MGI $^{2}$ & DT $^{3}$ & \\
\hline Matéria seca fecal (\%) & $24,79 \mathrm{a}$ & $24,81 \mathrm{a}$ & $20,81 \mathrm{a}$ & 36,74 \\
Consumo de amido (kg) & $3,15 \mathrm{a}$ & $3,32 \mathrm{a}$ & $2,73 \mathrm{a}$ & 27,45 \\
Amido fecal (\%) & $24,00 \mathrm{a}$ & $28,46 \mathrm{a}$ & $22,69 \mathrm{a}$ & 22,32 \\
FDN fecal (\%) & $46,29 \mathrm{a}$ & $35,65 \mathrm{~b}$ & $48,97 \mathrm{a}$ & 12,69 \\
pH fecal & $6,96 \mathrm{a}$ & $7,15 \mathrm{a}$ & $6,74 \mathrm{a}$ & 3,54 \\
Escore de consistência fecal & $3,12 \mathrm{~b}$ & $2,92 \mathrm{c}$ & $3,20 \mathrm{a}$ & 16,07 \\
Ganho médio diário, $\mathrm{kg} / \mathrm{dia}$ & $1,79 \mathrm{a}$ & $1,82 \mathrm{a}$ & $1,47 \mathrm{a}$ & 18,82 \\
Eficiência alimentar, $\mathrm{kg} / \mathrm{kg}$ & $0,197 \mathrm{a}$ & $0,246 \mathrm{a}$ & $0,219 \mathrm{a}$ & 15,65 \\
\hline
\end{tabular}

Médias seguidas de letras diferentes, na mesma linha, diferem $(\mathrm{P}<0,05)$ pelo teste Tukey. ${ }^{1}$ Dieta total mais bagaço de cana in natura; ${ }^{2}$ milho grão inteiro; ${ }^{3}$ dieta total.

De acordo com TURGEON et al. (1983), o amido fecal diminuiu linearmente conforme o aumento do nível da forragem na dieta. Segundo os autores, o decréscimo no consumo observado com o acréscimo do nível de forragem explica de certa forma, a queda do amido fecal. No presente trabalho não houve redução do amido fecal com o aumento da forragem na ração. A explicação dada pelo autor para a queda do amido fecal, em função da diminuição do consumo da MS da dieta, não corrobora os achados deste trabalho, já que nesta pesquisa houve aumento de consumo com o acréscimo de forragem na ração e não houve diferença na percentagem de amido fecal entre os tratamentos.

LEE et al. (1982) observaram em novilhos Hereford confinados em 112 dias que a percentagem de amido fecal foi maior à medida que a proporção de MGI aumentou na dieta em relação ao milho floculado a vapor. Em geral, houve uma tendência do $\mathrm{pH}$ fecal mais alto ser associado com baixo conteúdo de amido fecal. Portanto, $\mathrm{pH}$ fecal pode ser um indicador útil de avaliação do amido geral em ruminantes alimentados com dietas de alta proporção de concentrado, porém, não em todas as situações, já que GALYEAN et al. (1979) observaram, em dietas com diferentes tamanhos de partículas de milho, que o pH fecal não foi relacionado ao conteúdo de amido fecal. RUSSEL et al. (1980) relataram uma baixa correlação $(r=-0,35)$ entre $\mathrm{pH}$ fecal e a concentração de amido fecal em dietas com alta adição de MGI $(88,5 \%)$ com a inclusão de $0,9 \%$ de bicarbonato de sódio, $1,8 \%$ de calcário e a combinação dos dois.
WHEELER \& NOLLER (1977) concluíram que medidas de $\mathrm{pH}$ feitas em amostras de fezes são um excelente indicador do $\mathrm{pH}$ no intestino delgado. Baixo pH fecal está associado com grandes quantidades de amido nas fezes de bovinos alimentado com alta ração concentrada. Na mesma linha, DEGREGÓRIO et al. (1982) afirmaram que o $\mathrm{pH}$ é o mais simples indicador da quantidade de amido fermentado no intestino grosso, pois reflete o grau de acidez resultante dessa fermentação.

Os níveis de amido na ração refletem o padrão da dieta e a forma de processamento do grão. CAETANO (2008) evidenciou teores de amido fecal para o milho de $8,6 \%$ e MS fecal de $19,5 \%$ e amido fecal para o sorgo de $13,2 \%$ e MS fecal de $22,7 \%$ e medidas de $\mathrm{pH}$ fecal para o milho de 6,56 e para o sorgo de 6,07. Neste trabalho, os resultados de amido fecal (\% MS) foram superiores em $81,82 \%$ (amido fecal do sorgo), 230,93\% (amido fecal do milho) e $71,89 \%$ (amido fecal do sorgo) para os tratamentos DT+BIN, MGI e DT. O autor observou para MS fecal do sorgo valor de $22,7 \%$, o que está próximo do obtido neste trabalho para os tratamentos DT+BIN e DT. No entanto, o valor da MS fecal do milho está abaixo 27,23\% comparado ao tratamento MGI (Tabela 5).

As medidas de $\mathrm{pH}$ fecais reveladas neste estudo foram mais altas $14,66 \%$ para o tratamento DT+BIN, $11,04 \%$ para o tratamento DT e $8,99 \%$ para o tratamento MGI, do que os valores encontrados por (CAETANO, 2008). Provavelmente, as medidas mais elevadas do $\mathrm{pH}$ tenham ocorrido em função dos menores consumos de MS dos 
tratamentos. Esse fato está de acordo com LEDOUX et al. (1985) que afirmaram que, em consumo de MS de $1,7 \%$ do peso corporal, a ingestão do amido de milho não é alta o bastante para diminuir o $\mathrm{pH}$ fecal.

Possivelmente, maiores níveis de amido obtidos nas fezes dos bovinos dos tratamentos, comparados ao trabalho de CAETANO (2008), estejam relacionados com a maior ingestão de amido. DeGREGÓRIO et al. (1982) relataram que o não processamento do milho e a menor utilização do amido no trato gastrintestinal alcança maior teor de amido nas fezes. Além do mais, pode ser devido ao horário de coleta das fezes dos animais, que foi realizado no período da manhã. CAETANO (2008) constatou que há uma relação significativa entre o teor de amido fecal e o tempo após o trato dos animais. A variação do amido em função do tempo pode ser explicada com base no comportamento ingestivo dos animais e no manejo dos confinamentos, pois o autor demonstrou que o teor de amido fecal apresentou um comportamento, no tempo, descrito por uma curva polinomial. Essa curva demonstra maiores teores de amido nas fezes durante as primeiras horas após o fornecimento da dieta. Com o decorrer do tempo há uma queda no teor de amido, que dura cerca de 10 a 12 horas.

CAETANO (2008) observou também que o valor do amido fecal para o período da manhã foi de $7,5 \%$ da MS, enquanto o valor obtido para o período da tarde foi de 3,5\%. Logo, a diferença de $100 \%$ entre os teores da manhã e da tarde deve ser considerada como limitante na utilização desse indicador sem a padronização da coleta.

Assim, horário de coleta e maior quantidade de grão, e ainda, no caso do tratamento MGI, o não processamento do milho (Tabela 3) da dieta, obviamente podem disponibilizar maior teor de amido nas fezes. É importante enfatizar que CAETANO (2008) trabalhou com níveis mais baixos de amido, consistindo em $26,54 \%$ de grão de milho em rações com $81 \%$ de concentrado.

NUNES (2008) observou consumo de amido em rações com $73 \%$ de concentrado (52\% milho grão seco e $31,57 \%$ de amido) de $2,44 \mathrm{~kg} /$ dia e $91 \%$ de concentrado (70,25\% milho grão seco e $48,40 \%$ de amido) de 4,32 kg/dia. Para o amido fecal encontrou teores de 13,9 e $19,27 \%$ e para medidas de $\mathrm{pH}$ fecal 6,02 e 5,97, respectivamente. Relativamente ao consumo de amido (CAM), o teor de amido fecal (TAF) e pH fecal (pHF) da ração com $91 \%$ de concentrado obtido pelo autor foi $30,12 \%$ maior para CAM, 47,69\% menor para TAF e 19,77\% menor para pHF, quando confrontados com a ração MGI com $75 \%$ grão de milho inteiro e 54,62\% de amido. Assim, esse autor verificou que os animais do tratamento com $91 \%$ de concentrado apresentaram mais amido nas fezes, em função do menor aproveitamento de energia digestível em relação ao tratamento com $73 \%$ de concentrado, justificando que isto aconteceu em razão da utilização de bovinos Nelore no experimento. Neste trabalho alguns animais do tratamento MGI (Figura 1) apresentaram fezes com consistência líquida, denotando problemas de fermentação com sintomas de acidose.

Nesse contexto, NUNES (2008) observou que existe diferença quanto ao teor de amido nas fezes de animais de diferentes grupos genéticos, indicando que bovinos Nelore perdem $28 \%$ mais amido nas fezes que animal cruzado, o que confere a natureza dos animais.

PUTRINO et al. (2006) ressaltaram maiores consumos de MS para Nelore em dietas com 64,10\% de NDT, enquanto, que Brangus apresentaram consumo máximo de MS com 67,05\% de NDT na dieta. Nesse sentido, a argumentação de CAETANO (2008) de que bovino Nelore aproveita menos energia digestível pode ser uma justificativa plausível para o presente estudo, já que as dietas dos tratamentos DT+BIN, MG e DT apresentam NDT de $74,68 \%$ e $81,21 \%$ e 80,21 , valores bem acima dos observados por PUTRINO et al. (2006).

DEPENBUSCH et al. (2008) verificaram, em 251 amostras fecais de novilhos suplementados com dietas ( $81 \%$ milho laminado seco), valores médios de amido fecal de $23 \%$, com uma variação mínima de 1,2\% até um máximo de 59,6\%, com desvio padrão de mais de $11 \%$. ZINN et al. (2007) compilaram dados de concentração de amido de 32 estudos de metabolismo e mostraram valores médios de amido fecal de 5,9\% com uma ampla variação de 0 a $44 \%$. Portanto, os teores médios de amidos fecais observados neste trabalho são consistentes, uma vez que esses autores, também trabalharam com dietas com $90 \%$ de concentrados e os valores aqui obtidos estão próximos aos desses autores.

GOROCICA-BUENFIL \& LOERCH (2005) enfatizaram o aumento de $45 \%$ na concentração de amido fecal em bovinos com alimentação de milho integral comparado ao milho triturado. $\mathrm{O}$ que não foi constatado neste trabalho, apesar de o amido das dietas dos tratamentos ter advindo de fontes distintas de grãos (Tabela 3) e de diferentes formas de processamento. IRELANPPERRY \& STALLINGS (1993) concluíram que vacas leiteiras alimentadas com dietas de pouca forragem têm $\mathrm{pH}$ fecal menor, amido fecal mais alto e menor escore de consistência de fezes. Desse modo, vacas que consomem dietas com baixa proporção de forragem exibem maior consumo de matéria seca, que pode resultar em uma taxa de passagem mais rápida, originando com isto o aumento na perda de amido pelo rúmen. Contudo, o amido que não é digerido no intestino delgado ou 
fermentado no intestino grosso passa para as fezes. Por outro lado, vacas que ingerem dietas de alta proporção de forragem apresentam baixo consumo de amido e taxa de passagem mais lenta ocasionando à dieta maior digestibilidade e redução na perda de amido no trato gastrointestinal mais baixo e nas fezes. Como resultado, a fermentação no trato mais baixo pode ser diminuída, resultando em maior $\mathrm{pH}$ fecal. Assim, das variáveis citadas anteriormente, a característica de escore de consistência de fezes, menor para o tratamento MGI, está de acordo com o autor.

A avaliação das fezes de bovinos pode fornecer valiosa informação relativa ao local e extensão da digestão e fermentação de alimentos consumidos. Normalmente, a maioria do alimento ingerido pelo bovino é degradada no rúmen e a maior parte dos nutrientes absorvidos ocorre no rúmen ou intestino delgado. Quando o alimento não é oportunamente fermentado no rúmen, alguns nutrientes não degradados podem alcançar o intestino delgado e ser absorvidos, porém, se a quantidade é também excessiva, a taxa de passagem é rápida e nutrientes podem escapar da digestão e absorção no intestino delgado. Quando ração rigorosamente carente em fibra ou também alta em carboidrato não estrutural (CNE) é fornecida para os bovinos, a fermentação no intestino grosso pode ser abrangente, resultando em efeitos negativos na saúde e produção de bovinos (KONONOFF et al., 2002).

A consistência das fezes em bovinos depende amplamente do conteúdo de água e é uma função do teor de umidade e quantidade de tempo que o alimento permanece no trato digestivo do animal. $\mathrm{O}$ material fecal normal tem uma consistência semelhante a um mingau e forma uma cúpula abobodada amontoada com 25,4 a $50,8 \mathrm{~mm}$ de altura (KONONOFF et al., 2002).

$\mathrm{O}$ menor teor de MS fecal do tratamento MGI deste ensaio em relação ao observado por CAETANO (2008), demonstrado pelo escore de consistência das fezes (Tabela 5), presumivelmente, é causado pelo baixo teor de FDNfe (Tabela 4) da dieta MGI, menores consumos de FDNfe e de FDN (IRELANPPERRY \& STALLINGS, 1993).

A dieta MGI com menor teor de FDNfe apresentou menor escore de consistência de fezes e menor percentagem de FDN fecal. Porém, a percentagem de amido e $\mathrm{pH}$ fecal, MS fecal e o consumo do amido não foram afetados pelo teor de FDNfe nas rações. Os dados apresentados (Tabela 5) corroboram em parte os achados de IRELANPPERRY \& STALLINGS (1993), que verificaram FDN fecal menor e escore fecal maior em vacas consumindo dieta de baixa proporção de forragem. Por outro lado, ao contrário dos resultados desses autores, dietas totalmente concentradas e de baixa proporção de forragem não influenciaram na percentagem de amido fecal, MS fecal, $\mathrm{pH}$ fecal e consumo do amido.

A concentração de FDN fecal foi igual para os tratamentos DT+BIN e DT. O menor teor de FDN fecal no tratamento MGI é proporcionado pelo menor consumo de FDN dos animais e, possivelmente, pela maior digestibilidade dessa dieta.

Os animais do tratamento MGI apresentaram fezes com consistência mais mole, com o valor de escore de 2,92 (Tabela 5). As fezes dos bovinos do tratamento DT+BIN foram de consistência mais firme com valor de escore de 3,12, considerada normal, e a dos animais do tratamento DT foram de consistência mais dura com valor de escore de 3,20.

IRELANPPERRY \& STALLINGS (1993) salientaram que vacas que consomem dietas de baixa proporção de forragem apresentam fezes que, visualmente, parecem ser de consistência mais líquida. Assim, de acordo com esse autor, vacas que ingerem dieta de alta forragem, porém ingerem mais fibra e menos MS, excretam fezes que recebem escore de consistência visual mais alta (dura).

A consistência amolecida para as fezes no tratamento MGI (Figura 1), possivelmente, aconteceu em função do menor teor de fibra e a maior concentração de amido na dieta (Tabela 4).

Os tratamentos DT+BIN e DT embora tenham apresentado níveis de FDNfe próximos, apresentaram consistência das fezes diferentes. Provavelmente, a consistência das fezes mais dura para o tratamento DT ocorreu por causa da distribuição e diferenças no tamanho de partícula, conforme relatado por HEINRICHS \& KONONOFF (2002), e fonte de fibra, que propiciam uma maior seleção de alimentos pelos animais. Em acréscimo, esse tratamento contém subprodutos de fontes de fibra de não forragem (FFNF) com menores tamanhos de partículas, que reduzem a motilidade ruminal, conforme reportado por FURLAN et al. (2006), e atuam com menor efetividade do que a forragem (ARMENTANO \& PEREIRA, 1999), que, somada à menor ingestão da dieta por esses animais, reflete na formação de fezes mais duras em função da menor taxa de passagem.

A consistência firme das fezes para o tratamento DT+BIN pode ter ocorrido em função do maior teor de fibra em relação ao tratamento MGI e o uso de fonte de forragem que promove uma melhor efetividade da fibra, promovendo uma taxa de passagem mais lenta.

Observam-se na Tabela 6 as correlações dos teores de amido nas fezes com $\mathrm{pH}$ fecal, ganho médio diário e eficiência alimentar. 


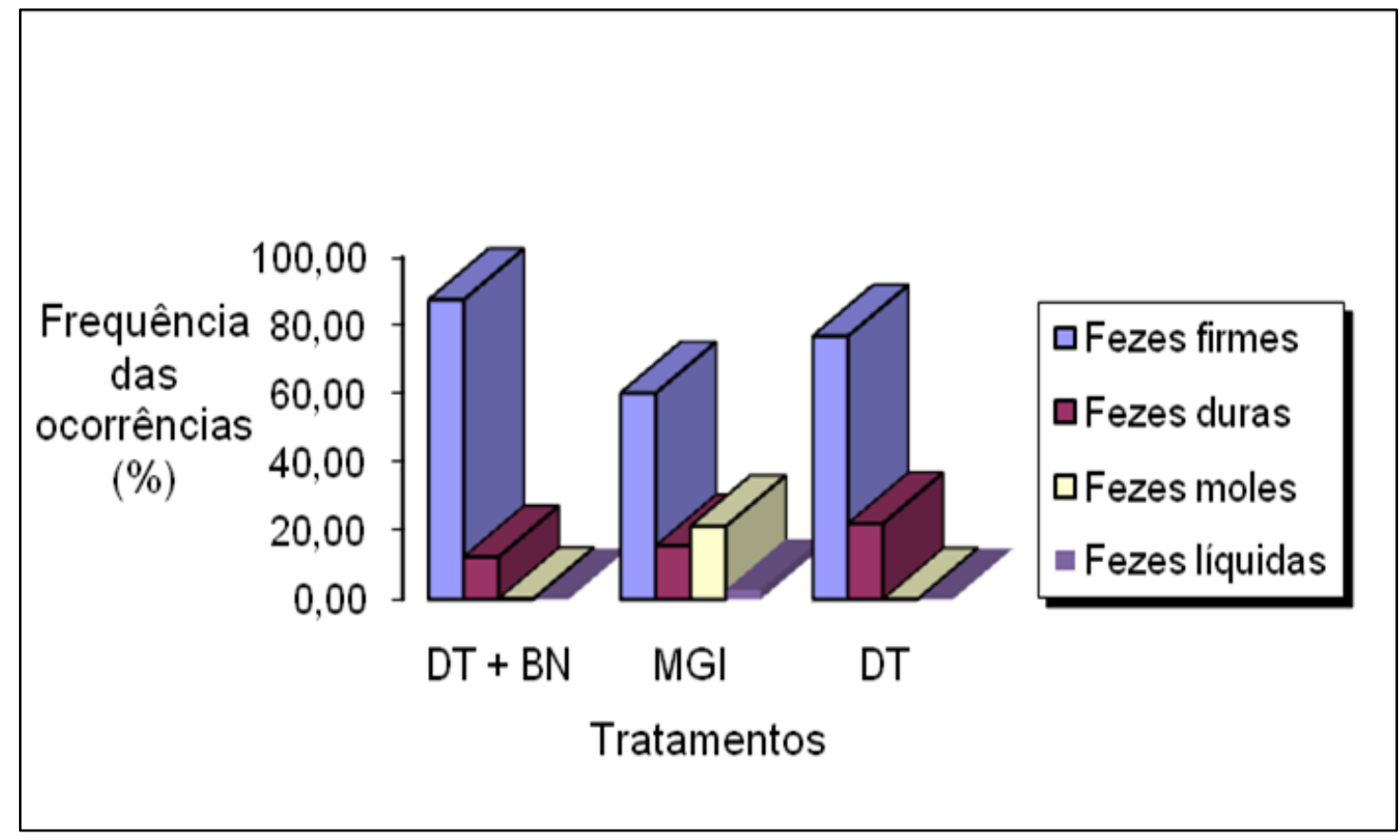

FIGURA 1. Características de fezes de Nelore em confinamento, alimentados com dietas de alta proporção de concentrado.

TABELA 6 - Correlações entre o teor de amido fecal e pH fecal, ganho médio diário, eficiência alimentar com seus respectivos coeficientes de correlação e níveis de probabilidade

\begin{tabular}{lcc}
\hline \multicolumn{1}{c}{ Correlação } & Coeficiente de correlação & $\mathrm{P}$ \\
\hline Amido fecal x pH fecal & 0,10 & 0,4 \\
Amido fecal x Ganho médio diário & 0,36 & 0,1 \\
Amido fecal x Eficiência alimentar & 0,48 & $<0,03$ \\
\hline
\end{tabular}

Não houve correlação entre o $\mathrm{pH}$ fecal $(\mathrm{P}>0,01)$ com o teor de amido nas fezes. Portanto, o presente resultado está em desacordo com WHEELER \& NOLLER (1977), TURGEON et al. (1983) LEDOUX et al. (1985), IRELANPPERRY \& STALLINGS (1993), MARUTA \& ORTOLANY, (2002), CHANNON et al. (2004), CAETANO (2008), NUNES (2008) e DEPENBUSCH et al. (2008), pois esses autores observaram relação negativa entre o $\mathrm{pH}$ fecal e o amido nas fezes de bovinos.

CHANNON et al. (2004) demonstraram correlação entre as variáveis de amido fecal e ganho médio diário. Anteriormente, LEDOUX et al. (1985) obtiveram correlação positiva baixa entre a percentagem de amido nas fezes e ganho médio diário. TURGEON et al. (1983), CAETANO (2008) e NUNES (2008) não observaram correlações entre amido fecal e ganho de peso. No presente estudo não foi constatada correlação entre as variáveis de amido fecal e o ganho médio diário $(\mathrm{P}=0,10)$. Todavia evidenciou-se a existência de correlação entre amido fecal e eficiência alimentar $(\mathrm{P}<0,03)$ (Tabela 6), o que sugere que o consumo do amido pode estar relacionado ao desempenho do animal. STELLA (2010) constatou que não houve correlação entre amido fecal e eficiência alimentar. $\mathrm{O}$ autor concluiu que, em dieta com teor de amido relativamente baixo, os parâmetros fecais não foram indicativos de a eficiência alimentar.

As estimativas de correlação entre características de ganho médio diário (GMD), consumo de matéria seca (CMS), consumo de amido (CAM), consumo de fibra detergente neutro (CFDN), consumo de fibra detergente ácido (CFDA), percentagem de fibra detergente neutro fecal (FDNfe) com consistência de fezes dura, pastosa, mole e líquida encontram-se na Tabela 7. 
TABELA 7 - Correlações simples de valores de ganho médio diário (GMD), consumo de matéria seca (CMS), consumo de amido (CAM), consumo de fibra detergente neutro (CFDN), consumo de fibra detergente ácido (CFDA) e \% de fibra detergente neutro fecal (FDNF) com indicadores de consistência em fezes com características firmes (FF), duras (FD), moles (FM) e líquidas (FL)

\begin{tabular}{lcc}
\hline \multicolumn{1}{c}{ Correlação } & Coeficiente de correlação & $\mathrm{P}$ \\
\hline GMD x FD & $-0,53$ & 0,03 \\
CMS x FF & 0,68 & 0,0009 \\
CMS x FD & $-0,68$ & 0,0036 \\
CAM x FF & 0,54 & 0,013 \\
CAM x FD & $-0,81$ & 0,0001 \\
CFDN x FF & 0,69 & 0,0007 \\
CFDN x FD & $-0,58$ & 0,019 \\
CFDA x FF & 0,71 & 0,0004 \\
CFDA x FD & $-0,52$ & 0,036 \\
FDNF x FM & $-0,61$ & 0,06 \\
FDNF x FL & $-0,55$ & 0,012 \\
\hline
\end{tabular}

Das características estudadas, apenas a percentagem de FDN fecal com fezes mole não alcançou diferença significativa. O CMS, CAM, CFDN e CFDA obtiveram estimativa de correlação positiva com fezes de consistência firme. O GMD, CMS, CAM, CFDN e CFDA apresentaram estimativa de correlação negativa com fezes de consistência dura. A FDN fecal obteve estimativa de correlação negativa com fezes de consistência líquida.

O consumo de matéria seca foi positivamente correlacionado $(\mathrm{r}=0,68) \mathrm{com}$ a consistência de FF e negativamente $(r=-0,68)$ com a $\mathrm{FD}$, indicando que subprodutos de fontes de fibra de não forragem (FFNF), com menores tamanhos de partículas, reduzem a motilidade ruminal (FURLAN et al., 2006) e atua com menor efetividade do que a forragem, proporcionando menor ingestão de alimentos e refletindo na formação de fezes mais dura em função da menor taxa de passagem (ARMENTANO \& PEREIRA, 1999).

O consumo de amido foi correlacionado positivamente com a consistência de $\mathrm{FF}(\mathrm{r}=0,54) \mathrm{e}$ negativamente $(r=-0,81)$ com a FD, evidenciando que o maior consumo de amido favorece uma taxa de passagem mais rápida promovendo um menor escore de consistência de fezes. Os consumos de FDN ( $\mathrm{r}=$ $0,69)$ e FDA $(r=0,71)$ correlacionaram-se positivamente com a consistência de FF. Os consumos de FDN $(r=-0,58)$ e FDA $(r=-0,52)$ correlacionaram-se negativamente com a consistência de FD. Essas ocorrências demonstram que menor ingestão de fibra pode resultar em fezes de consistência de escore mais baixo. A FDN fecal
(FDNF) foi negativamente correlacionada $(\mathrm{r}=-55)$ com as fezes de consistência líquida, sustentando que a observação visual da perda da consistência fecal pode ser em função da ocorrência de mais amido e menos fibra nas fezes.

\section{CONCLUSÕES}

Dieta de alta proporção de concentrado com adição de $10 \%$ de bagaço in natura de cana-deaçúcar na matéria seca proporciona maior frequência de fezes com escore de consistência firme.

\section{REFERÊNCIAS}

ARMENTANO, L.; PEREIRA, M. Symposium: meeting the fiber requirements of dairy cows measuring the effectiveness of fiber by animal response trials. Journal of Dairy Science, Savoy, v. 80, n. 7, p. 1416-1425, 1997.

ASSOCIATION OF OFFICIAL ANALYTICAL CHEMISTS. Official methods of anlysis. $15^{\text {th }}$ ed. Washington D. C., 1990. 1141 p.

BRASIL. Ministério da Agricultura Pecuária e Abastecimento. Instituto Nacional de Meteorologia $-10^{\circ}$ Distrito de Meteorologia. Goiânia, Go, 2008.

CAETANO, M. Estudo das perdas de amido em confinamentos brasileiros e do uso do amido fecal como ferramenta de manejo de bovinos confinados. 2008. 76 f. Dissertação (Mestrado em Agronomia) Escola Superior de Agricultura Luiz de Queiroz, Piracicaba. Disponível em http://www.teses.usp.br/teses/disponiveis/11/11139/tde28072008-152702/publico/mariana.pdf 
CAMPOS, F. P.; NUSSIO, C. M. B.; NUSSIO, L. G. Métodos de análise de alimentos. Piracicaba: FEALQ, 2004. $135 \mathrm{p}$

CHANNON, A. F.; ROWE, J. B.; HERD, R. M. Genetic variation in starch digestion in feedlot cattle and its association with residual feed intake. Australian Journal of Experimental Agriculture, Collingwood, v. 44, n. 5, p. $469-474,2004$.

CNCPS. Cornell net carbohydrate and protein system Ithaca Cornell University, 2002. software. Version 5.0.18.

DeGREGORIO, R. M.; TUCKER, R. E.; MITCHELL, G. E. JR.; GILL, W. W. Carbohydrate fermentation in the large intestine of lambs. Journal of Animal Science, Savoy, 1982. v. 54, n. 4, p. 855-862. 1982.

DEPENBUSCH, B. E.; NAGARAJA, T. G.; SARGEANT, J. M.; DROUILLARD, J. S.; LOE, E. R.; CORRIGAN, M. E. Influence of processed grains on fecal $\mathrm{pH}$, starch concentration, and shedding of Escherichia coli O157 in feedlot cattle. Journal of Animal Science, Savoy, v. 86, n. 3, p. 632-639, 2008.

FURLAN, R. L.; MACARI, M.; FARIA FILHO, D. E. Anatomia e fisiologia do trato gastrintestinal. In: Berchielli, T. T.; Pires, A. V.; Oliveira, S. G. Nutrição de ruminantes. Jaboticabal: Funep, 2006. cap. 1, p. 1-23.

GALYEAN, M. L.; WAGNER, D. G.; OWENS, F. N. Corn particle size and site and extent of digestion by steers. Journal of Animal Science, Savoy, v. 49, n. 1, p.204-210, 1979

GOROCICA-BUENFIL, M. A.; LOERCH, S. C. Effect of cattle age, forage level, and corn processing on diet digestibility and feedlot performance. Journal of Animal Science, Savoy, v. 83, n. 3, p. 705-714, 2005.

HEINRICHS, J.; KONONOFF, P. Evaluating particle size of forages and TMRs usingthe New Penn State Forage Particle Separator. Pennsylvania: The PennsylvaniaState University, Departament of Dairy and Animal Science [online], 2002. Disponível em: http://www.das.psu.edu/das/pdf/separadordeparticulas.pdf /view?searchterm=Jud

20 Heinrichs\%20and\%20Paul\%20Kononoff. Acesso em: 20 jun. 2008.

HUNTINGTON, G. B.; HARMON, D. L.; RICHARDS, J. Sites, rates, and limits of starch digestion and glucose metabolism in growing cattle. Journal of Animal Science, Savoy, v. 84 (E. Suppl.), n. 13, p. E14-E24, 2006.

IRELANDPERRY, R. L.; STALLINGS, C. C. Fecal consistency as related to dietary composition in lactating holstein cows. Journal of Dairy Science, Savoy, v. 76, n. 4, p. 1074-1082, 1993.

KONONOFF, P. J.; HEINRICHS, A. J.; BUCKMASTER, D. R. Modification of the Penn State Forage and Total Mixed Ration Particle Separator and the Effects of Moisture Content on its Measurements. Journal of Dairy Science, Savoy, v. 86, n. 5, p. 1858-1863, 2003.
KONONOFF, P. J.; HEINRICHS, A. J.; VARGA, G. Using manure evaluation to enhance dairy cattle. Dairy and Animal Science. Pensilvania: Pennsylvania: The Pennsylvania State University, Departament of Dairy and Animal Science [online], 2002. Disponível em: http://www.das.psu.edu/dairy/dairy-nutrition/pdf-dairynutrition/manure.pdf/view?searchterm=Kononoff, \%20P. Acesso em: 08 fev. 2009.

LEDOUX , D. R.; WILLIAMS, J. E.; STROUD, T. E.; GARNER, G. B.; PATERSON, J. A. Influence of forage level on passage rate, digestibility and performance of cattle. Journal of Animal Science, Savoy, v. 61, n. 6, p. 1567-1575, 1985.

LEE, R. W.; GALYEAN, M. L.; LOFGREEN, G. P. Effects of mixing whole shelled and steam flaked corn in finishing diets on feedlot performance and site and extent of digestion in beef steers. Journal of Animal Science, Savoy, v. 55, n. 3, p. 475-483, 1982.

MARUTA, C. A.; ORTOLANI, E. L. Susceptibilidade de bovinos das raças Jersey e Gir à acidose láctica ruminal: I - variáveis ruminais e fecais. Ciência Rural, Santa Maria, v. 32, n. 1, p. 55-59, 2002.

MERTENS, D. R. Creating a system for meeting the fiber requirements of dairy cows. Journal of Dairy Science, Savoy, v. 80, n. 7, p. 1463-1481, 1997.

NATIONAL RESEARCH COUNCIL - NRC. Nutrients requeriments of beef cattle. 7 . ed. Washington, D. C., 1996. $232 \mathrm{p}$.

NUNES, A. J. C. Uso combinado de ionóforo e virginamicina em novilhos Nelore confinados com dietas de alto concentrado. 2008. 67 f. Dissertação (Mestrado em Agronomia) - Escola Superior de Agricultura Luiz de Queiroz, Piracicaba. Disponível em http://www.teses.usp.br/teses/disponiveis/11/11139/tde13102008-104631/pt-br.php

OWENS, F. N.; ZINN, R. A.; KIM, Y. K. Limits to starch digestion in the ruminant small intestine. Journal of Animal Science, Savoy, v. 63, n. 5, p. 1634-1648, 1986.

PUTRINO, S. M. P.; LEME, P. R.; SILVA, S. L.; ALLEONI, G. F.; LANNA, D. P. D., LIMA, C. G.; GROSSKLAUS, C. Exigências líquidas de proteína e energia para ganho de peso de tourinhos Brangus e Nelore alimentados com dietas contendo diferentes proporções de concentrado. Revista Brasileira de Zootecnia, Viçosa, v. 35, n. 1, p. 292-300, 2006.

ROBERTSON, J. B.; VAN SOEST, P. J. The detergent system of analysis. In: JAMES, W. P. T.; THEANDER, $O$. The analysis of dietary fibre in food. New York: Marcel Dekker, 1981. Chap.9, p.123-158.

RUSSELL, J. R.; YOUNG A. W.; JORGENSEN, N. A. Effect of sodium bicarbonate and limestone additions to high grain diets on feedlot performance and ruminal and fecal parameters in finishing steers. Journal of Animal Science, Savoy, v. 51, n. 1, p. 996-1002, 1980.

RUSSELL, J. R.; YOUNG, A. W.; JORGENSEN, N. A. 
Effect of dietary corn starch intake on pancreatic amylase and intestinal maltase and $\mathrm{pH}$ in cattle. Journal of Animal Science, Savoy, v. 52, n. 5, p. 1177-1182, 1981.

SAS. User`s guide: Statistics. Cary: Statistical Analysis System Institute, 2000.1686p.

SNIFFEN, C.J.; O’CONNOR, J.D.; VAN SOEST, P.J; FOX, D. G.; RUSSEL, J. B. A net carbohydrate and protein system for evaluating cattle diets: II. Carbohydrate and protein availability. Journal of Animal Science, Savoy, v. 70, n.11, p. 3562-3577, 1992.

STELLA, T. R. Desempenho, característica de carcaça e parâmetros fecais indicativos da digesta do amido e suas relações com a eficiência alimentar de bovinos de corte. 2010. 63 f. Dissertação (Mestrado) - Faculdade de Zootecnia e Engenharia de Alimentos, Pirassununga.
Disponível em http://www.teses.usp.br/teses/ disponiveis/74/74131/tde-21022011-113521/pt-br.php

TURGEON, O. A.; BRINK, JR. D. R.; R. A. BRITTON, R. A. Corn particle size mixtures, roughage level and starch utilization in finishing steer diets. Journal of Animal Science, Savoy, v. 57, n. 3, p. 739-749, 1983.

WHEELER, W. E.; NOLLER, C. H. Gastrointestinal tract $\mathrm{pH}$ and starch in feces of ruminants. Journal of Animal Science, Savoy, v. 44, n. 1, p. 131-135, 1977.

ZINN, R. A.; BARRERAS, A.; CORONA, L.; OWENS, F. N.; WARE, R. A. Starch digestion by feedlot cattle: predictions from analysis of feed and fecal starch and nitrogen. Journal of Animal Science, Savoy, 2007. v. 85, n. 7, p. 1727-1730, 2007.

Protocolado em:13 mar. 2009. Aceito em:28 fev. 2012 